International Journal of Pure and Applied Mathematics

Volume 97 No. $1 \quad 2014,49-66$

ISSN: 1311-8080 (printed version); ISSN: 1314-3395 (on-line version)

url: http://www.ijpam.eu

doi: http://dx.doi.org/10.12732/ijpam.v97i1.6

\title{
BOUNDEDNESS OF MARCINKIEWICZ INTEGRALS ON PRODUCT SPACES AND EXTRAPOLATION
}

\author{
Mohammed Ali ${ }^{1} \S$, Ameera Al-Senjlawi ${ }^{2}$ \\ ${ }^{1,2}$ Department of Mathematics and Statistics \\ Jordan University of Science and Technology \\ Irbid, JORDAN
}

\begin{abstract}
In this article, we establish $L^{p}$ estimates for the Marcinkiewicz integral operators with rough kernels on product spaces. These estimates and extrapolation arguments improve and extend some known results on Marcinkiewicz integrals.
\end{abstract}

AMS Subject Classification: 40B15, 40B20, 40B25

Key Words: $\quad L^{p}$ boundedness, Marcinkiewicz integrals, rough kernels, extrapolation

\section{Introduction}

Let $n, m \geq 2$, and let $\mathbf{S}^{N-1}(N=n$ or $m)$ be the unit sphere in $\mathbf{R}^{N}$ equipped with the normalized Lebesgue surface measure $d \sigma=d \sigma(\cdot)$. Also, let $x^{\prime}=x /|x|$ for $x \in \mathbf{R}^{n} \backslash\{0\}, y^{\prime}=y /|y|$ for $y \in \mathbf{R}^{m} \backslash\{0\}$. Let $p^{\prime}$ denote to the exponent conjugate to $p$.

For $\rho=a_{1}+i b_{1}, \tau=a_{2}+i b_{2}\left(a_{1}, b_{1}, a_{2}, b_{2} \in \mathbf{R}\right.$ with $\left.a_{1}, a_{2}>0\right)$, let $K_{\Omega, h}(x, y)=\Omega\left(x^{\prime}, y^{\prime}\right)|x|^{\rho-n}|y|^{\tau-m} h(|x|,|y|)$, where $h$ is a measurable function on $\mathbf{R}^{+} \times \mathbf{R}^{+}$and $\Omega$ is a function on $\mathbf{S}^{n-1} \times \mathbf{S}^{m-1}$ with $\Omega \in L^{1}\left(\mathbf{S}^{n-1} \times \mathbf{S}^{m-1}\right)$

Received: June 24, 2014

(c) 2014 Academic Publications, Ltd.

${ }^{\S}$ Correspondence author url: www.acadpubl.eu 
satisfying the cancellation conditions:

$$
\int_{\mathbf{S}^{n-1}} \Omega\left(x^{\prime}, .\right) d \sigma\left(x^{\prime}\right)=\int_{\mathbf{S}^{m-1}} \Omega\left(., y^{\prime}\right) d \sigma\left(y^{\prime}\right)=0
$$

For suitable mappings $\phi, \psi: \mathbf{R}^{+} \rightarrow \mathbf{R}$, a measurable function $h$ on $\mathbf{R}^{+} \times \mathbf{R}^{+}$ and an $\Omega$ on $\mathbf{S}^{n-1} \times \mathbf{S}^{m-1}$ satisfying (1), we define the Marcinkiewicz integral operator $\mathcal{M}_{\Omega, h, \phi, \psi}^{\rho, \tau}$ for $f \in \mathcal{S}\left(\mathbf{R}^{n} \times \mathbf{R}^{m}\right)$ by

$$
\mathcal{M}_{\Omega, h, \phi, \psi}^{\rho, \tau} f(x, y)=\left(\int_{0}^{\infty} \int_{0}^{\infty}\left|\frac{1}{t^{\rho} s^{\tau}} F_{t, s}^{\phi, \psi} f(x, y)\right|^{2} \frac{d t d s}{t s}\right)^{1 / 2}
$$

where

$$
F_{t, s}^{\phi, \psi} f(x, y)=\int_{|u| \leq t} \int_{|v| \leq s} f\left(x-\phi(|u|) u^{\prime}, y-\psi(|v|) v^{\prime}\right) K_{\Omega, h}(u, v) d u d v
$$

For simplicity, we denote $\mathcal{M}_{\Omega, h, \phi, \psi}^{\rho, \tau}$ by $\mathcal{M}_{\Omega, h}^{\rho, \tau}$ if $\phi(r)=\psi(r)=r$. Also, if $\rho=\tau=1$ and the function $h=1$, the operator $\mathcal{M}_{\Omega, h}^{\rho, \tau}$ is the classical Marcinkiewicz integral operator on product domains which is denoted by $\mathcal{M}_{\Omega, c}$.

The investigations of the $L^{p}\left(\mathbf{R}^{n} \times \mathbf{R}^{m}\right)$ boundedness of Marcinkiewicz integral operators on product spaces began by Ding in [17] in which he established the $L^{2}$ boundedness of $\mathcal{M}_{\Omega, c}$ if $\Omega \in L(\log L)^{2}\left(\mathbf{S}^{n-1} \times \mathbf{S}^{m-1}\right)$. Subsequently, it was studied by many mathematicians. For example, the author of [14] proved that $\mathcal{M}_{\Omega, c}$ is bounded for all $1<p<\infty$ provided that $\Omega \in L(\log L)^{2}\left(\mathbf{S}^{n-1} \times \mathbf{S}^{m-1}\right)$. For more information about the importance and the recent advances on the study of such operators, the readers are refereed (for instance to [1], [3], [13], [15], [16], [29], [30], as well as [31], and the references therein).

We point out that the study of parametric Marcinkiewicz integral operator was initiated by Hörmander in [23] in which he showed that $\mathcal{M}_{\Omega, 1}^{\rho}$ (in the one parameter case) is bounded on $L^{p}\left(\mathbf{R}^{n}\right)$ for $1<p<\infty$ when $\rho>0$ and $\Omega \in \operatorname{Lip}_{\alpha}\left(\mathbf{S}^{n-1}\right)$ with $\alpha>0$, and subsequently by Sakamoto and Yabuta in [24] ( for the corresponding results in the one parameter cases, see for instance [2], [5], [6], [8], [10], [11], [12], [18], [19], [26], and [28]).

For $d \neq 0$, we let $\mathcal{H}_{d}$ be the class of all functions $\phi:(0, \infty) \rightarrow \mathbf{R}$ which are smooth and satisfy the following growth conditions:

$$
|\phi(t)| \leq C_{1} t^{d}, \quad\left|\phi^{\prime \prime}(t)\right| \leq C_{2} t^{d-2}, \quad C_{3} t^{d-1} \leq\left|\phi^{\prime}(t)\right| \leq C_{4} t^{d-1}
$$


for $t \in(0, \infty)$, and $C_{1}, C_{2}, C_{3}$ plus $C_{4}$ are positive constants independent of $t$. Also, for $\gamma \geq 1$, we let $\Delta_{\gamma}\left(\mathbf{R}^{+} \times \mathbf{R}^{+}\right)$denote the collection of all measurable functions $h: \mathbf{R}^{+} \times \mathbf{R}^{+} \rightarrow \mathbf{C}$ satisfying

$$
\sup _{R_{1}, R_{2}>0}\left(\frac{1}{R_{1} R_{2}} \int_{0}^{R_{1}} \int_{0}^{R_{2}}|h(t, s)|^{\gamma} d t d s\right)^{1 / \gamma}<\infty .
$$

The primary focus of this paper is establishing $L^{p}$ estimates of $\mathcal{M}_{\Omega, h, \phi, \psi}^{\rho, \tau}$ for various functions $\phi \in \mathcal{H}_{d_{1}}, \psi \in \mathcal{H}_{d_{2}}$ for some $d_{1}, d_{2} \neq 0$, and $h \in \Delta_{\gamma}\left(\mathbf{R}^{+} \times \mathbf{R}^{+}\right)$; and then apply the extrapolation argument used in [4] to obtain new improved results. Our main result is formulated as follows:

Theorem 1. Let $\Omega \in L^{q}\left(\mathbf{S}^{n-1} \times \mathbf{S}^{m-1}\right)$ for some $1<q \leq 2, h \in$ $\Delta_{\gamma}\left(\mathbf{R}^{+} \times \mathbf{R}^{+}\right)$for some $\gamma>1$. Suppose that $\phi \in \mathcal{H}_{d_{1}}, \psi \in \mathcal{H}_{d_{2}}$ for some $d_{1}, d_{2} \neq 0$. Then there exists a constant $C_{p}$ (independent of $\Omega, h, \gamma$, and $q$ ) such that

$$
\begin{aligned}
& \left\|\mathcal{M}_{\Omega, h, \phi, \psi}^{\rho, \tau} f\right\|_{L^{p}\left(\mathbf{R}^{n} \times \mathbf{R}^{m}\right)} \\
& \quad \leq C_{p} \frac{A(\gamma)}{q-1}\|h\|_{\Delta_{\gamma}\left(\mathbf{R}^{+} \times \mathbf{R}^{+}\right)}\|\Omega\|_{L^{q}\left(\mathbf{S}^{n-1} \times \mathbf{S}^{m-1}\right)}\|f\|_{L^{p}\left(\mathbf{R}^{n} \times \mathbf{R}^{m}\right)}
\end{aligned}
$$

for $|1 / p-1 / 2|<\min \left\{1 / 2,1 / \gamma^{\prime}\right\}$, where

$$
A(\gamma)= \begin{cases}\gamma & \text { if } \gamma>2 \\ (\gamma-1)^{-1} & \text { if } 1<\gamma \leq 2\end{cases}
$$

The power of our theorem lies in using its conclusion and extrapolation (see [4]) to obtain improved results. In particular, Theorem 1 and extrapolation lead to the following theorem.

Theorem 2. Suppose that $h \in \Delta_{\gamma}\left(\mathbf{R}^{+} \times \mathbf{R}^{+}\right)$for some $\gamma>1$, and $\Omega$ satisfies (1). Let $\phi \in \mathcal{H}_{d_{1}}$ and $\psi \in \mathcal{H}_{d_{2}}$ for some $d_{1}, d_{2} \neq 0$.

(i) If $\Omega \in B_{q}^{(0,0)}\left(\mathbf{S}^{n-1} \times \mathbf{S}^{m-1}\right)$ for some $q>1$, then

$$
\begin{aligned}
& \left\|\mathcal{M}_{\Omega, h, \phi, \psi}^{\rho, \tau} f\right\|_{L^{p}\left(\mathbf{R}^{n} \times \mathbf{R}^{m}\right)} \\
& \quad \leq C_{p} A(\gamma)\|h\|_{\Delta_{\gamma}\left(\mathbf{R}^{+} \times \mathbf{R}^{+}\right)}\|f\|_{L^{p}\left(\mathbf{R}^{n} \times \mathbf{R}^{m}\right)}\left(1+\|\Omega\|_{B_{q}^{(0,0)}\left(\mathbf{S}^{n-1} \times \mathbf{S}^{m-1}\right)}\right)
\end{aligned}
$$


for $|1 / p-1 / 2|<\min \left\{1 / 2,1 / \gamma^{\prime}\right\}$

(ii) If $\Omega \in L(\log L)\left(\mathbf{S}^{n-1} \times \mathbf{S}^{m-1}\right)$, then

$$
\begin{aligned}
& \left\|\mathcal{M}_{\Omega, h, \phi, \psi}^{\rho, \tau} f\right\|_{L^{p}\left(\mathbf{R}^{n} \times \mathbf{R}^{m}\right)} \\
& \quad \leq C_{p} A(\gamma)\|h\|_{\Delta_{\gamma}\left(\mathbf{R}^{+} \times \mathbf{R}^{+}\right)}\|f\|_{L^{p}\left(\mathbf{R}^{n} \times \mathbf{R}^{m}\right)}\left(1+\|\Omega\|_{L(\log L)\left(\mathbf{S}^{n-1} \times \mathbf{S}^{m-1}\right)}\right)
\end{aligned}
$$

for $|1 / p-1 / 2|<\min \left\{1 / 2,1 / \gamma^{\prime}\right\}$.

Remarks. (1) The class $\mathcal{H}_{d}$ was introduced by Fan and Pan in [21] in their studies of singular integrals. Model functions for the $\phi \in \mathcal{H}_{d}$ are $\phi(t)=t^{d}$ with $d>0$ or $\phi(t)=t^{r}$ with $r<0$. We point out that the class $\mathcal{H}_{d}$ is empty when $d=0$.

(2) The authors of [3] established the $L^{p}(1<p<\infty)$ boundedness of $\mathcal{M}_{\Omega, c}^{1,1}$ under the condition $\Omega \in L(\log L)\left(\mathbf{S}^{n-1} \times \mathbf{S}^{m-1}\right)$. Furthermore, they proved that the exponent 1 is optimal for the $L^{2}$ boundedness of $\mathcal{M}_{\Omega, c}^{1,1}$ to hold.

(3) Al-Qassem in [1] showed that $\mathcal{M}_{\Omega, h}^{1,1}$ is of type $(p, p)$ for $p \in(1, \infty)$ if $\Omega \in B_{q}^{(0,0)}\left(\mathbf{S}^{n-1} \times \mathbf{S}^{m-1}\right)$ and $h \in L^{\infty}\left(\mathbf{R}^{+} \times \mathbf{R}^{+}\right)$. Moreover, in the same paper Al-Qassem showed that the condition $\Omega \in B_{q}^{(0,0)}\left(\mathbf{S}^{n-1} \times \mathbf{S}^{m-1}\right)$ is optimal in the sense that there exists $\Omega \in B_{q}^{(0, \nu)}\left(\mathbf{S}^{n-1} \times \mathbf{S}^{m-1}\right)$ for all $-1<\nu<0$ such that $\mathcal{M}_{\Omega, c}$ is not bounded in $L^{2}\left(\mathbf{R}^{n} \times \mathbf{R}^{m}\right)$.

(4) If $\Omega$ belongs to the block space $B_{q}^{(0,0)}\left(\mathbf{S}^{n-1} \times \mathbf{S}^{m-1}\right)$ and $h \in \Delta_{\gamma}\left(\mathbf{R}^{+} \times \mathbf{R}^{+}\right)$ for some $q, \gamma>1$, then the $L^{p}$ boundedness of $\mathcal{M}_{\Omega, \phi, \psi, h}^{\rho, \tau}$ was obtained in [9] for any $p$ satisfying $|1 / p-1 / 2|<\min \left\{1 / 2,1 / \gamma^{\prime}\right\}$ provided that $\phi, \psi \in \mathcal{H}_{d}$ for some $d \neq 0$.

(5) In the one parameter case, the authors of [10] used the extrapolation arguments to show that if $\Omega$ belongs to the class $L(\log L)^{1 / 2}\left(\mathbf{S}^{n-1}\right)$ or to the class $B_{q}^{(0,-1 / 2)}\left(\mathbf{S}^{n-1}\right), h \in \Delta_{\gamma}\left(\mathbf{R}^{+}\right)$, and $\phi \in \mathcal{H}_{d}$ for some $q, \gamma>1, d \neq 0$, then $\mathcal{M}_{\Omega, \phi, h}^{\rho}$ is bounded on $L^{p}\left(\mathbf{R}^{n}\right)$ for any $p$ satisfying $|1 / p-1 / 2|<\min \left\{1 / 2,1 / \gamma^{\prime}\right\}$.

Here and henceforth, the letter $\mathrm{C}$ denotes a bounded positive constant that may vary at each occurrence but independent of the essential variables. 


\section{Preliminary Results}

In this section, we introduce some notations and give some auxiliary lemmas used in the sequel. For suitable functions $\phi, \psi$ on $\mathbf{R}^{+}$, a measurable function $h: \mathbf{R}^{+} \times \mathbf{R}^{+} \rightarrow \mathbf{C}, \theta \geq 2$, and $\Omega: \mathbf{S}^{n-1} \times \mathbf{S}^{m-1} \rightarrow \mathbf{R}$, we define the family of measures $\left\{\sigma_{\Omega, \phi, \psi, h, t, s}: t, s \in \mathbf{R}^{+}\right\}$and the corresponding maximal operators $\sigma_{\Omega, \phi, \psi, h}^{*}$ and $M_{\Omega, \phi, \psi, h, \theta}$ on $\mathbf{R}^{n} \times \mathbf{R}^{m}$ by

$$
\begin{gathered}
\int_{\mathbf{R}^{n} \times \mathbf{R}^{m}} f d \sigma_{\Omega, \phi, \psi, h, t, s} \\
=t^{-\rho} s^{-\tau} \int_{1 / 2 t \leq|u| \leq t} \int_{1 / 2 s \leq|v| \leq s} f\left(\phi(|u|) u^{\prime}, \psi(|v|) v^{\prime}\right) K_{\Omega, h}(u, v) d u d v, \\
\sigma_{\Omega, \phi, \psi, h}^{*} f(x, y)=\sup _{t, s \in \mathbf{R}^{+}}|| \sigma_{\Omega, \phi, \psi, h, t, s}|* f(x, y)|,
\end{gathered}
$$

and

$$
M_{\Omega, \phi, \psi, h, \theta} f(x, y)=\sup _{i, j \in \mathbf{Z}} \int_{\theta^{i}}^{\theta^{i+1}} \int_{\theta^{j}}^{\theta^{j+1}}|| \sigma_{\Omega, \phi, \psi, h, t, s}|*| f(x, y) \mid \frac{d t d s}{t s},
$$

where $\left|\sigma_{\Omega, \phi, h, t}\right|$ is defined in the same way as $\sigma_{\Omega, \phi, h, t}$, but with replacing $K_{\Omega, h}$ by $\left|K_{\Omega, h}\right|$. We write $t^{ \pm \alpha}=\min \left\{t^{\alpha}, t^{-\alpha}\right\}$ and $\left\|\sigma_{\Omega, \phi, \psi, h, t, s}\right\|$ for the total variation of $\sigma_{\Omega, \phi, \psi, h, t, s}$.

The following lemma can be obtained by applying a similar argument used in $[7]$.

Lemma 3. Let $\Omega \in L^{q}\left(\mathbf{S}^{n-1} \times \mathbf{S}^{m-1}\right)$ for some $q>1$ and satisfies the cancellation conditions (1). Suppose that $\phi \in \mathcal{H}_{d_{1}}$ and $\psi \in \mathcal{H}_{d_{2}}$ for some $d_{1}, d_{2} \neq 0$. For $t, s>0$, let

$$
G_{t, s}(r, k)=\int_{\mathbf{S}^{n-1} \times \mathbf{S}^{m-1}} e^{-i\{\phi(t r) x \cdot \xi+\psi(s k) y \cdot \eta\}} \Omega(x, y) d \sigma(x) d \sigma(y) .
$$

Then there are constants $C$ and $\alpha$ with $0<\alpha<\frac{1}{2 q^{\prime}}$ such that

$$
\int_{1 / 2}^{1} \int_{1 / 2}^{1}\left|G_{t, s}(r, k)\right|^{2} \frac{d r d k}{r k} \leq C\left|\xi t^{d_{1}}\right|^{ \pm \frac{\alpha}{q^{\prime}}}\left|\eta s^{d_{2}}\right|^{ \pm \frac{\alpha}{q^{\prime}}}\|\Omega\|_{L^{q}\left(\mathbf{S}^{n-1} \times \mathbf{S}^{m-1}\right)}^{2} .
$$


Proof. We prove this lemma only for $d_{1}, d_{2}>0$ because the proof for the other cases are essentially the same and requires only minor modifications. Also we prove this lemma for the case $1<q \leq 2$, since $L^{q}\left(\mathbf{S}^{n-1} \times \mathbf{S}^{m-1}\right) \subseteq$ $L^{2}\left(\mathbf{S}^{n-1} \times \mathbf{S}^{m-1}\right)$ for $q \geq 2$. By Schwarz inequality, we get that

$$
\begin{aligned}
& \int_{1 / 2}^{1} \int_{1 / 2}^{1}\left|G_{t, s}(r, k)\right|^{2} \frac{d r d k}{r k} \\
& \quad \leq C \int_{\mathbf{S}^{m-1}}\left(\int_{\mathbf{S}^{n-1} \times \mathbf{S}^{n-1}} J(\xi, x, u) \Omega(x, y) \overline{\Omega(u, y)} d \sigma(x) d \sigma(u)\right)^{d \sigma(y),}
\end{aligned}
$$

where $J(\xi, x, u)=\int_{1 / 2}^{1} e^{-i \phi(t r) \xi \cdot(x-u)} \frac{d r}{r}$. Write $J(\xi, x, u)=\int_{1 / 2}^{1} Y_{t}^{\prime}(r) \frac{d r}{r}$, where

$$
Y_{t}(r)=\int_{1 / 2}^{r} e^{-i \phi(t z) \xi \cdot(x-u)} d z, 1 / 2 \leq z \leq r \leq 1
$$

By Van der Corput's lemma, the assumptions on $\phi$ and integration by parts, we conclude

$$
|J(\xi, x, u)| \leq C\left|\xi \cdot(x-u) t^{d_{1}}\right|^{-1},
$$

which when combined with the trivial estimate $|J(\xi, x, u)| \leq C$ leads to

$$
|J(\xi, x, u)| \leq C\left|\xi t^{d_{1}}\right|^{-\alpha}\left|\xi^{\prime} \cdot(x-u)\right|^{-\alpha}
$$

for any $0<\alpha<1$. Thus, by Hölder's inequality we have

$$
\begin{aligned}
\int_{1 / 2}^{1} \int_{1 / 2}^{1}\left|G_{t, s}(r, k)\right|^{2} \frac{d r d k}{r k} & \leq C\left|\xi t^{d_{1}}\right|^{-\frac{\alpha}{q^{\prime}}}\|\Omega\|_{L^{q}\left(\mathbf{S}^{n-1} \times \mathbf{S}^{m-1}\right)}^{2} \\
& \times\left(\int_{\mathbf{S}^{n-1} \times \mathbf{S}^{n-1}}\left|\xi^{\prime} \cdot(x-u)\right|^{-\alpha q^{\prime}} d \sigma(x) d \sigma(y)\right)^{\prime}
\end{aligned}
$$

By choosing $0<2 \alpha q^{\prime}<1$, we get that the last integral is finite, and hence

$$
\int_{1 / 2}^{1} \int_{1 / 2}^{1}\left|G_{t, s}(r, k)\right|^{2} \frac{d r d k}{r k} \leq C\left|\xi t^{d_{1}}\right|^{-\frac{\alpha}{q^{\prime}}}\|\Omega\|_{L^{q}\left(\mathbf{S}^{n-1} \times \mathbf{S}^{m-1}\right)}^{2} .
$$


In the same manner, we obtain

$$
\int_{1 / 2}^{1} \int_{1 / 2}^{1}\left|G_{t, s}(r, k)\right|^{2} \frac{d r d k}{r k} \leq C\left|\eta s^{d_{2}}\right|^{-\frac{\alpha}{q^{\prime}}}\|\Omega\|_{L^{q}\left(\mathbf{S}^{n-1} \times \mathbf{S}^{m-1}\right)}^{2} .
$$

Using the cancelation property of $\Omega$, we have by a simple change of variable that

$$
\begin{aligned}
& \int_{1 / 2}^{1} \int_{1 / 2}^{1}\left|G_{t, s}(r, k)\right|^{2} \frac{d r d k}{r k} \\
& \leq C \int_{1 / 2}^{1} \int_{1 / 2}^{1}\left(\int_{\mathbf{S}^{n-1} \times \mathbf{S}^{m-1}}\left|e^{-i \phi(t r) \xi \cdot x}-1\right||\Omega(x, y)| d \sigma(x) d \sigma(y)\right)^{2} \frac{d r d k}{r k} \\
& \leq C|\xi|^{2}\|\Omega\|_{L^{1}\left(\mathbf{S}^{n-1} \times \mathbf{S}^{m-1}\right)}^{2} \int_{\frac{1}{2}}^{1}|\phi(t r)|^{2} \frac{d r}{r} .
\end{aligned}
$$

Since $|\phi(t r)| \leq C(t r)^{d_{1}}$ and $\frac{1}{2}<r<1$, we achieve

$$
\int_{1 / 2}^{1} \int_{1 / 2}^{1}\left|G_{t, s}(r, k)\right|^{2} \frac{d r d k}{r k} \leq C\left|\xi t^{d_{1}}\right|^{2}\|\Omega\|_{L^{1}\left(\mathbf{S}^{n-1} \times \mathbf{S}^{m-1}\right)}^{2} .
$$

Combine the last estimate with the trivial estimate $\int^{1} \int^{1}\left|G_{t, s}(r, k)\right|^{2} \frac{d r d k}{r k} \leq$ $C\|\Omega\|_{L^{1}\left(\mathbf{S}^{n-1} \times \mathbf{S}^{m-1}\right)}^{2}$, we derive

$$
\int_{1 / 2}^{1} \int_{1 / 2}^{1}\left|G_{t, s}(r, k)\right|^{2} \frac{d r d k}{r k} \leq C\left|\xi t^{d_{1}}\right|^{\frac{\alpha}{q^{2}}}\|\Omega\|_{L^{q}\left(\mathbf{S}^{n-1} \times \mathbf{S}^{m-1}\right)}^{2} .
$$

Similarly, we obtain that

$$
\int_{1 / 2}^{1} \int_{1 / 2}^{1}\left|G_{t, s}(r, k)\right|^{2} \frac{d r d k}{r k} \leq C\left|\eta s^{d_{2}}\right|^{\frac{\alpha}{q^{\prime}}}\|\Omega\|_{L^{q}\left(\mathbf{S}^{n-1} \times \mathbf{S}^{m-1}\right)}^{2} .
$$

Therefore, by (4)-(7), the proof of this lemma is complete 
Lemma 4. Let $\theta \geq 2, \Omega \in L^{q}\left(\mathbf{S}^{n-1} \times \mathbf{S}^{m-1}\right)$ for some $q>1$ and $h \in$ $\Delta_{\gamma}\left(\mathbf{R}^{+} \times \mathbf{R}^{+}\right)$for some $\gamma>1$. Suppose that $\phi, \psi$ are given as in Lemma 3 . Then there are constants $C$ and $\alpha$ with $0<\alpha<\frac{1}{2 q^{\prime}}$ such that

$$
\begin{aligned}
& \left\|\sigma_{\Omega, \phi, \psi, h, t, s}\right\| \leq C\|h\|_{\Delta_{\gamma}\left(\mathbf{R}^{+} \times \mathbf{R}^{+}\right)}\|\Omega\|_{L^{q}\left(\mathbf{S}^{n-1} \times \mathbf{S}^{m-1}\right)} ; \\
& \theta^{i+1} \theta^{j+1} \\
& \int_{\theta^{i}} \int_{\theta^{j}}\left|\hat{\sigma}_{\Omega, \phi, \psi, h, t, s}(\xi, \eta)\right|^{2} \frac{d t d s}{t s} \leq C \ln ^{2}(\theta)\|h\|_{\Delta_{\gamma}\left(\mathbf{R}^{+} \times \mathbf{R}^{+}\right)}^{2}\|\Omega\|_{L^{q}\left(\mathbf{S}^{n-1} \times \mathbf{S}^{m-1}\right)}^{2} \\
& \times\left|\xi \theta^{j d_{1}}\right|^{ \pm \frac{\alpha}{\omega q^{\prime}}}\left|\eta \theta^{i d_{2}}\right|^{ \pm \frac{\alpha}{\omega q^{\prime}}}
\end{aligned}
$$

hold for all $i, j \in \mathbf{Z}$, where $\omega=\max \left\{2, \gamma^{\prime}\right\}$. The constant $C$ is independent of $i, j, \xi, \eta, q$, and $\theta$.

Proof. By using the definition of $\sigma_{\Omega, \phi, \psi, h, t, s}$, it is easy to show that (8) holds. By Hölder's inequality, we have that

$$
\begin{aligned}
\left|\hat{\sigma}_{\Omega, \phi, \psi, h, t, s}(\xi, \eta)\right| & \leq \int_{1 / 2}^{1} \int_{1 / 2}^{1}|h(t r, k s)| \int_{\mathbf{S}^{n-1} \times \mathbf{S}^{m-1}} e^{-i\{\phi(t r) x \cdot \xi+\psi(s k) y \cdot \eta\}} \\
& \times \Omega(x, y) d \sigma(x) d \sigma(y) \mid \frac{d r d k}{r k} \\
& \leq C\|h\|_{\Delta_{\gamma}\left(\mathbf{R}^{+} \times \mathbf{R}^{+}\right)}\left(\int_{1 / 2}^{1} \int_{1 / 2}^{1}\left|G_{t, s}(r, k)\right|^{\gamma^{\prime}} \frac{d r d k}{r k}\right)^{1 / \gamma^{\prime}}
\end{aligned}
$$

If $1<\gamma \leq 2$, then we conclude

$$
\begin{aligned}
& \left|\hat{\sigma}_{\Omega, \phi, \psi, h, t, s}(\xi, \eta)\right| \\
& \quad \leq\|h\|_{\Delta_{\gamma}\left(\mathbf{R}^{+} \times \mathbf{R}^{+}\right)}\|\Omega\|_{L^{1}\left(\mathbf{S}^{n-1} \times \mathbf{S}^{m-1}\right)}^{\left(1-2 / \gamma^{\prime}\right)}\left(\int_{1 / 2}^{1} \int_{1 / 2}^{1}\left|G_{t, s}(r, k)\right|^{2} \frac{d r d k}{r k}\right)^{1 / \gamma^{\prime}},
\end{aligned}
$$

and if $\gamma>2$, then by Hölder's inequality, we deduce

$$
\left|\hat{\sigma}_{\Omega, \phi, \psi, h, t, s}(\xi, \eta)\right| \leq\|h\|_{\Delta_{\gamma}\left(\mathbf{R}^{+} \times \mathbf{R}^{+}\right)}\left(\int_{1 / 2}^{1} \int_{1 / 2}^{1}\left|G_{t, s}(r, k)\right|^{2} \frac{d r d k}{r k}\right)^{1 / 2}
$$


Thus, in either case we reach

$$
\begin{aligned}
& \left|\hat{\sigma}_{\Omega, \phi, \psi, h, t, s}(\xi, \eta)\right| \\
& \leq C\|h\|_{\Delta_{\gamma}\left(\mathbf{R}^{+} \times \mathbf{R}^{+}\right)}\|\Omega\|_{L^{1}\left(\mathbf{S}^{n-1} \times \mathbf{S}^{m-1}\right)}^{(\omega-2) / \gamma^{\prime}}\left(\int_{1 / 2}^{1} \int_{1 / 2}^{1}\left|G_{t, s}(r, k)\right|^{2} \frac{d r d k}{r k}\right)^{1 / \omega},
\end{aligned}
$$

where $\omega=\max \left\{2, \gamma^{\prime}\right\}$; hence, by Lemma 3 we obtain $\left|\hat{\sigma}_{\Omega, \phi, \psi, h, t, s}(\xi, \eta)\right|^{2} \leq C\|h\|_{\Delta_{\gamma}\left(\mathbf{R}^{+} \times \mathbf{R}^{+}\right)}^{2}\|\Omega\|_{L^{q}\left(\mathbf{S}^{n-1} \times \mathbf{S}^{m-1}\right)}^{2}\left|\xi t^{d_{1}}\right|^{ \pm \frac{2 \alpha}{\omega q^{\prime}}}\left|\eta s^{d_{2}}\right|^{ \pm \frac{2 \alpha}{\omega q^{\prime}}}$.

Therefore,

$$
\begin{aligned}
& \theta^{i+1} \theta^{j+1} \\
& \int_{\theta^{i}} \int_{\theta^{j}}\left|\hat{\sigma}_{\Omega, \phi, \psi, h, t, s}(\xi, \eta)\right|^{2} \frac{d t d s}{t s} \leq C \ln ^{2}(\theta)\|h\|_{\Delta_{\gamma}\left(\mathbf{R}^{+} \times \mathbf{R}^{+}\right)}^{2}\|\Omega\|_{L^{q}\left(\mathbf{S}^{n-1} \times \mathbf{S}^{m-1}\right)}^{2} \\
& \times\left|\xi \theta^{j d_{1}}\right|^{ \pm \frac{\alpha}{\omega q^{\prime}}}\left|\xi \theta^{i d_{2}}\right|^{ \pm \frac{\alpha}{\omega q^{\prime}}} .
\end{aligned}
$$

The following lemma follows immediately by applying a well known argument found in [20].

Lemma 5. Let $\varphi \in \mathcal{H}_{d}$ for some $d \neq 0$. Define the maximal function

$$
\mathcal{M}_{\varphi} f(x)=\sup _{t \in \mathbf{R}^{+}} \frac{1}{t}\left|\int_{\frac{1}{2} t}^{t} f(x-\varphi(r)) d r\right| .
$$

Then for $1<p \leq \infty$, there exists a constant $C_{p}$ such that

$$
\left\|\mathcal{M}_{\varphi}(f)\right\|_{L^{p}(\boldsymbol{R})} \leq C_{p}\|f\|_{L^{p}(\boldsymbol{R})}
$$

for any $f \in L^{p}(\mathbf{R})$.

Proof. It is easy to see that $\mathcal{M}_{\varphi} f(x) \leq C \sup _{k \in \mathbf{Z}}\left|\int_{2^{k}}^{2^{k+1}} f(x-\varphi(r)) \frac{d r}{r}\right|$. Define a sequence of measures $\nu_{k}$ on $\mathbf{R}$ by

$$
\hat{\nu}_{k}(\xi)=\int_{2^{k}}^{2^{k+1}} e^{-i \varphi(r) \xi} \frac{d r}{r} .
$$


Following the same approaches used in the proof of Lemma 4, we achieve that

$$
\left\{\begin{array}{c}
\left|\hat{\nu}_{k}(\xi)\right| \leq C \\
\left|\hat{\nu}_{k}(\xi)-\hat{\nu}_{k}(0)\right| \leq C\left|2^{k d} \xi\right| \\
\left|\hat{\nu}_{k}(\xi)\right| \leq C\left|2^{k d} \xi\right|^{-1}
\end{array}\right.
$$

Therefore, by invoking Theorem A of [20] we conclude that $\mathcal{M}_{\varphi}(f)$ is bounded on $L^{p}(\mathbf{R})$ for $1<p \leq \infty$.

By Lemma 5 and using the same arguments as in [[25], Proposition 1 (pp. 72)] (se also [[21], Lemma 3.1]), we immediately get the following lemma.

Lemma 6. Let $\varphi \in \mathcal{H}_{d}$ for some $d \neq 0$ and $u \in \boldsymbol{S}^{N-1}$. Define the maximal function

$$
\mathcal{M}_{\phi, u} f(x)=\sup _{t \in \boldsymbol{R}^{+}} \frac{1}{t}\left|\int_{\frac{1}{2} t}^{t} f(x-\varphi(r) u) d r\right| .
$$

Then, a positive constant $C_{p}$ exists such that for any $f \in L^{p}\left(\mathbf{R}^{N}\right)$ with $1<p \leq$ $\infty$, we have

$$
\left\|\mathcal{M}_{\varphi, u}(f)\right\|_{L^{p}\left(\boldsymbol{R}^{N}\right)} \leq C_{p}\|f\|_{L^{p}\left(\boldsymbol{R}^{N}\right)}
$$

Lemma 7. Let $\Omega \in L^{1}\left(\mathbf{S}^{n-1} \times \mathbf{S}^{m-1}\right)$ and $h \in \Delta_{\gamma}\left(\mathbf{R}^{+} \times \mathbf{R}^{+}\right)$for some $\gamma>1$. Assume that and $\phi, \psi$ are given as in Theorem 1 . Then for any $f \in$ $L^{p}\left(\mathbf{R}^{n} \times \mathbf{R}^{m}\right)$ with $\gamma^{\prime}<p \leq \infty$, there exists a constant $C_{p}$ such that

$$
\left\|\sigma_{\Omega, \phi, \psi, h}^{*}(f)\right\|_{L^{p}\left(\mathbf{R}^{n} \times \mathbf{R}^{m}\right)} \leq C_{p}\|h\|_{\Delta_{\gamma}\left(\mathbf{R}^{+} \times \mathbf{R}^{+}\right)}\|\Omega\|_{L^{1}\left(\mathbf{S}^{n-1} \times \mathbf{S}^{m-1}\right)}\|f\|_{L^{p}\left(\mathbf{R}^{n} \times \mathbf{R}^{m}\right)} .
$$

Proof. By Hölder's inequality, we have

$$
\begin{aligned}
& \| \sigma_{\Omega, \phi, \psi, h}|* f(x, y)| \\
& \leq C\|h\|_{\Delta_{\gamma}\left(\mathbf{R}^{+} \times \mathbf{R}^{+}\right)}\|\Omega\|_{L^{1}\left(\mathbf{S}^{n-1} \times \mathbf{S}^{m-1}\right)}^{1 / \gamma}\left(\frac{1}{t s} \int_{\frac{t}{2}}^{t} \int_{\frac{s}{2}}^{s} \int_{\mathbf{S}^{n-1} \times \mathbf{S}^{m-1}}|\Omega(u, v)|\right. \\
& \left.\times|f(x-\phi(r) u, y-\psi(k) v)|^{\gamma^{\prime}} d \sigma(u) d \sigma(v) d r d k\right)^{1 / \gamma^{\prime}} .
\end{aligned}
$$

Hence, Minkowski's inequality for integrals yields that

$$
\left\|\sigma_{\Omega, \phi, \psi, h}^{*} f\right\|_{L^{p}\left(\mathbf{R}^{n} \times \mathbf{R}^{m}\right)} \leq\|h\|_{\Delta_{\gamma}\left(\mathbf{R}^{+} \times \mathbf{R}^{+}\right)}\|\Omega\|_{L^{1}\left(\mathbf{S}^{n-1} \times \mathbf{S}^{m-1}\right)}^{1 / \gamma}
$$




$$
\begin{aligned}
& \times\left(\int_{\mathbf{S}^{n-1} \times \mathbf{S}^{m-1}}|\Omega(u, v)|\right. \\
& \left.\times\left\|\left(\mathcal{M}_{\psi, v} \circ \mathcal{M}_{\phi, u}\right)\left(|f|^{\gamma^{\prime}}\right)\right\|_{L^{\left(p / \gamma^{\prime}\right)}\left(\mathbf{R}^{n} \times \mathbf{R}^{m}\right)} d \sigma(u) d \sigma(v)\right)^{1 / \gamma^{\prime}} \\
& \leq\|h\|_{\Delta_{\gamma}\left(\mathbf{R}^{+} \times \mathbf{R}^{+}\right)}\|\Omega\|_{L^{1}\left(\mathbf{S}^{n-1} \times \mathbf{S}^{m-1}\right)}\left\|\mathcal{M}_{\psi, v} \circ \mathcal{M}_{\phi, u}(|f|)\right\|_{L^{p}\left(\mathbf{R}^{n} \times \mathbf{R}^{m}\right)},
\end{aligned}
$$

where $\mathcal{M}_{\phi, u} f(x, y)=\mathcal{M}_{\phi, u} f(\cdot, y)(x), \mathcal{M}_{\psi, v} f(x, y)=\mathcal{M}_{\psi, v} f(x, \cdot)(y)$ and $\circ$ denotes the composition of operators. Thus, by using the last inequality and Lemma 6, we finish the proof of this lemma

The following lemma can be obtained by applying the arguments (with only minor modifications) used in [4] and [7].

Lemma 8. Let $h \in \Delta_{\gamma}\left(\mathbf{R}^{+} \times \mathbf{R}^{+}\right)$for some $\gamma>1, \Omega \in L^{q}\left(\mathbf{S}^{n-1} \times \mathbf{S}^{m-1}\right)$ for some $1<q \leq 2$ and $\theta=2^{q^{\prime} \gamma^{\prime}}$. Assume that $\phi, \psi$ are given as in Theorem 1 . Then for any $p$ satisfying $|1 / p-1 / 2|<\min \left\{1 / 2,1 / \gamma^{\prime}\right\}$, there exists a positive constant $C_{p}$ such that

$$
\begin{aligned}
& \left\|\left(\sum_{i, j \in \mathbf{Z}} \int_{\theta^{j}}^{\theta^{j+1}} \int_{\theta^{i}}^{\theta^{i+1}}\left|\sigma_{\Omega, \phi, \psi, h, t, s} * g_{i, j}\right|^{2} \frac{d s d t}{s t}\right)\right\|_{L^{p}\left(\mathbf{R}^{n} \times \mathbf{R}^{m}\right)} \\
& \leq C_{p} \frac{A(\gamma)}{q-1}\|h\|_{\Delta_{\gamma}\left(\mathbf{R}^{+} \times \mathbf{R}^{+}\right)}\|\Omega\|_{L^{q}\left(\mathbf{S}^{n-1} \times \mathbf{S}^{m-1}\right)} \|\left(\sum_{i, j \in \mathbf{Z}}\left|g_{i, j}\right|^{2} \|_{L^{p}\left(\mathbf{R}^{n} \times \mathbf{R}^{m}\right)}^{1 / 2}\right.
\end{aligned}
$$

holds for arbitrary functions $\left\{g_{i, j}(\cdot, \cdot), i, j \in \mathbf{Z}\right\}$ on $\mathbf{R}^{n} \times \mathbf{R}^{m}$.

Proof. Let us first prove this lemma for the case $1<\gamma \leq 2$. On one hand, if $2 \leq p<\frac{2 \gamma}{2-\gamma}$, then by duality, there is a non-negative function $\Lambda \in$ $L^{(p / 2)^{\prime}}\left(\mathbf{R}^{n} \times \mathbf{R}^{m}\right)$ with $\|\Lambda\|_{L^{(p / 2)^{\prime}}\left(\mathbf{R}^{n} \times \mathbf{R}^{m}\right)} \leq 1$ such that

$$
\|\left(\sum_{i, j \in \mathbf{Z}} \int_{\theta^{j}}^{\theta^{j+1}} \int_{\theta^{i}}^{\theta^{i+1}}\left|\sigma_{\Omega, \phi, \psi, h, t, s} * g_{i, j}\right|^{2} \frac{d t d s}{t s} \mid \|_{L^{p}\left(\mathbf{R}^{n} \times \mathbf{R}^{m}\right)}^{1 / 2}\right.
$$




$$
=\int_{\mathbf{R}^{n} \times \mathbf{R}^{m}} \sum_{i, j \in \mathbf{Z}} \int_{\theta^{j}}^{\theta^{j+1}} \int_{\theta^{i}}^{\theta^{i+1}}\left|\sigma_{\Omega, \phi, \psi, h, t, s} * g_{i, j}(x, y)\right|^{2} \frac{d t d s}{t s} \Lambda(x, y) d x d y .
$$

By Schwarz's inequality, we obtain

$$
\begin{array}{r}
\left|\sigma_{\Omega, \phi, \psi, h, t, s} * g_{i, j}\right|^{2} \leq C\|h\|_{\Delta_{\gamma}\left(\mathbf{R}^{+} \times \mathbf{R}^{+}\right)}^{\gamma}\|\Omega\|_{L^{q}\left(\mathbf{S}^{n-1} \times \mathbf{S}^{n-1}\right)} \\
\left(\left.\int_{\frac{1}{2} t{ }_{\frac{1}{2} s}}^{t} \int_{\mathbf{S}^{n-1} \times \mathbf{S}^{m-1}} \int_{i, j}(x-\phi(r) u, y-\psi(k) v)\right|^{2} \mid\right. \\
\left.\Omega(u, v)|h(r, k)|^{2-\gamma} d \sigma(u) d \sigma(v) \frac{d r d k}{r k}\right) .
\end{array}
$$

Thus, by a change of variable we derive that

$$
\begin{aligned}
& \|\left(\sum_{i, j \in \mathbf{Z}} \int_{\theta^{j}}^{\theta^{j+1}} \int_{\theta^{i}}^{\theta^{i+1}}\left|\sigma_{\Omega, \phi, \psi, h, t, s} * g_{i, j}\right|^{2} \frac{d t d s}{t s}\left\|^{1 / 2}\right\|_{L^{p}\left(\mathbf{R}^{n} \times \mathbf{R}^{m}\right)}^{2} \leq C\|h\|_{\Delta_{\gamma}\left(\mathbf{R}^{+} \times \mathbf{R}^{+}\right)}^{\gamma}\right.
\end{aligned}
$$

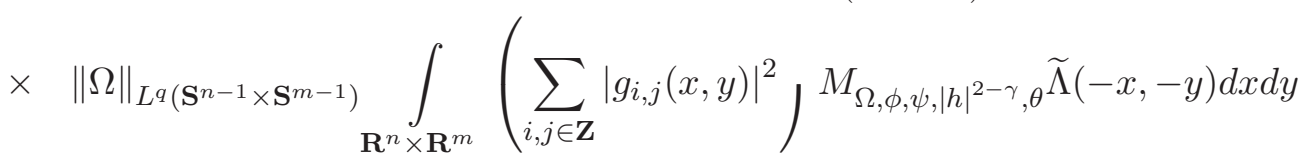

$$
\begin{aligned}
& \leq C\|h\|_{\Delta_{\gamma}\left(\mathbf{R}^{+} \times \mathbf{R}^{+}\right)}^{\gamma}\|\Omega\|_{L^{q}\left(\mathbf{S}^{n-1} \times \mathbf{S}^{m-1}\right)}\left\|\sum_{i, j \in \mathbf{Z}}\left|g_{i, j}\right|^{2}\right\|_{L^{(p / 2)}\left(\mathbf{R}^{n} \times \mathbf{R}^{m}\right)} \\
& \left\|M_{\Omega, \phi, \psi,|h|^{2-\gamma}, \theta} \widetilde{\Lambda}\right\|_{L^{(p / 2)^{\prime}}\left(\mathbf{R}^{n} \times \mathbf{R}^{m}\right)},
\end{aligned}
$$

where $\widetilde{\Lambda}(-x,-y)=\Lambda(x, y)$. As $h \in \Delta_{\gamma}\left(\mathbf{R}^{+} \times \mathbf{R}^{+}\right)$, we have $|h|^{2-\gamma} \in \Delta_{\frac{\gamma}{2-\gamma}}\left(\mathbf{R}^{+} \times\right.$ $\mathbf{R}^{+}$), and since $\left(\frac{p}{2}\right)^{\prime}>\left(\frac{\gamma}{2-\gamma}\right)^{\prime}$, then by Hölder's inequality and Lemma 7 , we reach that

$$
\begin{aligned}
& \left\|\left(\sum_{i, j \in \mathbf{Z}} \int_{\theta^{j}} \int_{\theta^{i}}^{\theta^{+1}}\left|\sigma_{\Omega, \phi, \psi, h, t, s} * g_{i, j}\right|^{2} \frac{d t d s}{t s}\right)^{1 / 2}\right\|_{L^{p}\left(\mathbf{R}^{n} \times \mathbf{R}^{m}\right)}^{2} \\
\leq & C \ln ^{2}(\theta)\|h\|_{\Delta_{\gamma}\left(\mathbf{R}^{+} \times \mathbf{R}^{+}\right)}^{\gamma}\|\Omega\|_{L^{q}\left(\mathbf{S}^{n-1} \times \mathbf{S}^{m-1}\right)} \|\left(\sum_{i, j \in \mathbf{Z}}\left|g_{i, j}\right|^{2}\left\|^{2}\right\|_{L^{p}\left(\mathbf{R}^{n} \times \mathbf{R}^{m}\right)}^{2}\right.
\end{aligned}
$$




$$
\begin{aligned}
& \times \quad\left\|\sigma_{\Omega, \phi, \psi,|h|^{2-\gamma}}^{*} \widetilde{\Lambda}\right\|_{L^{(p / 2)^{\prime}}\left(\mathbf{R}^{n} \times \mathbf{R}^{m}\right)} \\
& \leq C_{p} \frac{1}{[(\gamma-1)(q-1)]^{2}}\|h\|_{\Delta_{\gamma}\left(\mathbf{R}^{+} \times \mathbf{R}^{+}\right)}^{2}\|\Omega\|_{L^{q}\left(\mathbf{S}^{n-1} \times \mathbf{S}^{m-1}\right)}^{2} \\
& \\
& \left\|\sum_{i, j \in \mathbf{Z}}\left|g_{i, j}\right|^{2}\right\|_{L^{p}\left(\mathbf{R}^{n} \times \mathbf{R}^{m}\right)}^{1 / 2} \|^{2} .
\end{aligned}
$$

On the other hand, if $\frac{2 \gamma}{3 \gamma-2}<p<2$, by the duality, there are functions $\zeta=\zeta_{i, j}(x, y, t, s)$ defined on $\mathbf{R}^{n} \times \mathbf{R}^{m} \times \mathbf{R}^{+} \times \mathbf{R}^{+}$with

$$
\|\|\left\|\zeta_{i, j}\right\|_{L^{2}\left(\left[\theta^{i}, \theta^{i+1}\right] \times\left[\theta^{j}, \theta^{j+1}\right], \frac{d t d s}{t s}\right)}\left\|_{l^{2}}\right\|_{L^{p^{\prime}}\left(\mathbf{R}^{n} \times \mathbf{R}^{m}\right)} \leq 1
$$

such that

$$
\begin{aligned}
& \|\left(\sum_{i, j \in \mathbf{Z}} \int_{\theta^{j}} \int_{\theta^{i}}^{\theta^{j+1}}\left|\sigma_{\Omega, \phi, \psi, h, t, s} * g_{i, j}\right|^{2} \frac{d t d s}{t s} \mid\left\|_{L^{p}\left(\mathbf{R}^{n} \times \mathbf{R}^{m}\right)}^{1 / 2}\right\|_{\theta^{j+1}} \theta^{i+1}\right. \\
= & \int_{\mathbf{R}^{n} \times \mathbf{R}^{m}} \sum_{i, j \in \mathbf{Z}} \int_{\theta^{j}} \int_{\theta^{i}}\left(\sigma_{\Omega, \phi, \psi, h, t, s} * g_{i, j}(x, y)\right) \zeta_{i, j}(x, y, t, s) \frac{d t d s}{t s} d x d y \\
\leq & C_{p} \ln ^{2}(\theta)\left\|(\Upsilon(\zeta))^{1 / 2}\right\|_{L^{p^{\prime}}\left(\mathbf{R}^{n} \times \mathbf{R}^{m}\right)} \|\left(\sum_{i, j \in \mathbf{Z}}\left|g_{i, j}\right|^{2} \|_{L^{p}\left(\mathbf{R}^{n} \times \mathbf{R}^{m}\right)},\left(1 / 2 \|_{(1)}\right.\right.
\end{aligned}
$$

where

$$
\Upsilon(\zeta)(x, y)=\sum_{i, j \in \mathbf{Z}} \int_{\theta^{j}}^{\theta^{j+1}} \int_{\theta^{i}}^{\theta^{i+1}}\left|\sigma_{\Omega, \phi, \psi, h, t, s} * \zeta_{i, j}(x, y, t, s)\right|^{2} \frac{d t d s}{t s} .
$$

Since $\frac{p^{\prime}}{2}>1$, then there exists a function $\vartheta \in L^{\left(p^{\prime} / 2\right)^{\prime}}\left(\mathbf{R}^{n} \times \mathbf{R}^{m}\right)$ with

$$
\|\vartheta\|_{L^{\left(p^{\prime} / 2\right)^{\prime}}\left(\mathbf{R}^{n} \times \mathbf{R}^{m}\right)} \leq 1
$$

such that

$$
\left\|(\Upsilon(\zeta))^{1 / 2}\right\|_{L^{p^{\prime}}\left(\mathbf{R}^{n} \times \mathbf{R}^{m}\right)}^{2}
$$




$$
\begin{aligned}
& =\sum_{i, j \in \mathbf{Z}_{\mathbf{R}^{n} \times \mathbf{R}^{m}}} \int_{\theta^{j}} \int_{\theta^{i}}^{\theta^{j+1}} \int_{\theta^{i+1}}\left|\sigma_{\Omega, \phi, \psi, h, t, s} * \zeta_{i, j}(x, y, t, s)\right|^{2} \frac{d t d s}{t s} \vartheta(x, y) d x d y \\
& \leq C\|h\|_{\Delta_{\gamma}\left(\mathbf{R}^{+} \times \mathbf{R}^{+}\right)}^{\gamma}\|\Omega\|_{L^{q}\left(\mathbf{S}^{n-1} \times \mathbf{S}^{m-1}\right)}\left\|\sigma_{\Omega, \phi, \psi,|h|^{2-\gamma}, \theta}^{*}(\vartheta)\right\|_{L^{\left(p^{\prime} / 2\right)^{\prime}}\left(\mathbf{R}^{n} \times \mathbf{R}^{m}\right)} \\
& \times \|\left(\sum_{i, j \in \mathbf{Z}} \int_{\theta^{j}}^{\theta^{j+1}} \int_{\theta^{i}}^{\theta^{i+1}}\left|\zeta_{i, j}(\cdot, \cdot, t, s)\right|^{2} \frac{d t d s}{t s} \|_{L^{\left(p^{\prime} / 2\right)}\left(\mathbf{R}^{n} \times \mathbf{R}^{m}\right)}\right. \\
& \leq C\|h\|_{\Delta_{\gamma}\left(\mathbf{R}^{+} \times \mathbf{R}^{+}\right)}^{2}\|\Omega\|_{L^{q}\left(\mathbf{S}^{n-1} \times \mathbf{S}^{m-1}\right)}^{2} .
\end{aligned}
$$

Thus, by the last inequality plus $(10)$, and since $\ln (\theta) \leq \frac{C}{(\gamma-1)(q-1)}$, our estimate holds for $\frac{2 \gamma}{3 \gamma-2} \leq p<2$. using the same above technique gives the conclusion of Lemma 8 for the case $\gamma \geq 2$. Therefore, the desired estimate is satisfied.

\section{Proof of the Main Result}

We prove Theorem 1 by applying the same approaches that Al-Qassem and AlSalman [2] as well as Fan and Pan [22] used. Assume that $h \in \Delta_{\gamma}\left(\mathbf{R}^{+} \times \mathbf{R}^{+}\right)$ for some $\gamma>1$; and $\phi \in \mathcal{H}_{d_{1}}, \psi \in \mathcal{H}_{d_{2}}$ for some $d_{1}, d_{2} \neq 0$. By Minkowski's inequality, we get that

$$
\begin{aligned}
& \mathcal{M}_{\Omega, h, \phi, \psi}^{\rho, \tau} f(x, y)=\left(\int_{\mathbf{R}^{+} \times \mathbf{R}^{+}} \mid \sum_{i, j=0}^{\infty} \frac{1}{t^{\rho} s^{\tau}} \int_{2^{-i-1} t<|u| \leq 2^{-i} t} \int_{2^{-j-1} s<|v| \leq 2^{-j} s}\right. \\
\times & \left.\left.f\left(x-\phi(|u|) u^{\prime}, y-\psi(|v|) v^{\prime}\right) K_{\Omega, h}(u, v) d u d v\right|^{2} \frac{d t d s}{t s}\right)^{1 / 2} \\
\leq & \sum_{i, j=0}^{\infty}\left(\int_{\mathbf{R}^{+} \times \mathbf{R}^{+}} \mid \frac{1}{t^{\rho} s^{\tau}} \int_{2^{-i-1} t<|u| \leq 2^{-i} t} \int_{2^{-j-1} s<|v| \leq 2^{-j} s}\right. \\
\times & \left.\left.f\left(x-\phi(|u|) u^{\prime}, y-\psi(|v|) v^{\prime}\right) K_{\Omega, h}(u, v) d u d v\right|^{2} \frac{d t d s}{t s}\right)^{1 / 2} \\
\leq & \frac{2^{a_{1}+a_{2}}}{\left(2^{a_{1}}-1\right)\left(2^{a_{2}}-1\right)}\left(\int_{\mathbf{R}^{+} \times \mathbf{R}^{+}}\left|\sigma_{\Omega, \phi, \psi, h, t, s} * f(x, y)\right|^{2} \frac{d t d s}{t s}\right)^{1 / 2} .
\end{aligned}
$$

Let $\theta=2^{q^{\prime} \gamma^{\prime}}$, and for $i \in \mathbf{Z}$, let $\left\{\Gamma_{i, d_{1}}\right\}_{-\infty}^{\infty}$ be a smooth partition of unity in $(0, \infty)$ adapted to the interval $\mathcal{I}_{i, d_{1}}=\left[\theta^{-i d_{1}-\left|d_{1}\right|}, \theta^{-i d_{1}+\left|d_{1}\right|}\right]$. More precisely, 
we require the following:

$$
\begin{aligned}
\Gamma_{i, d_{1}} & \in C^{\infty}, 0 \leq \Gamma_{i, d_{1}} \leq 1, \sum_{i} \Gamma_{i, d_{1}}(t)=1, \\
\operatorname{supp} \Gamma_{i, d_{1}} & \subseteq \mathcal{I}_{i, d_{1}}, \text { and }\left|\frac{d^{k} \Gamma_{i, d_{1}}(t)}{d t^{k}}\right| \leq \frac{C_{k}}{t^{k}}
\end{aligned}
$$

where $C_{k}$ is independent of $\theta$. Define the multiplier operators $M_{i, j}$ on $\mathbf{R}^{n} \times \mathbf{R}^{m}$ by $\left(\widehat{M_{i, j} f}\right)(\xi, \eta)=\Gamma_{i, d_{1}}(|\xi|) \Gamma_{j, d_{2}}(|\eta|) \hat{f}(\xi, \eta)$. Then for any $f \in \mathcal{S}\left(\mathbf{R}^{n} \times \mathbf{R}^{m}\right)$ and $i, j \in \mathbf{Z}$, we have $f(x, y)=\sum_{d, l \in \mathbf{Z}} M_{i+d, j+l}(f)(x, y)$. Therefore, by Minkowski's inequality we obtain

$$
\mathcal{M}_{\Omega, h, \phi, \psi}^{\rho, \tau} f(x, y) \leq C \sum_{d, l \in \mathbf{Z}} S_{d, l} f(x, y)
$$

where

$$
\begin{gathered}
S_{d, l} f(x, y)=\left(\int_{0}^{\infty} \int_{0}^{\infty}\left|Y_{d, l}(x, y, t, s)\right|^{2} \frac{d t d s}{t s}\right)^{1 / 2}, \\
Y_{d, l}(x, y, t, s)=\sum_{i, j \in \mathbf{Z}} \sigma_{\Omega, \phi, \psi, h, t, s} * M_{i+d, j+l} f(x, y) \chi_{\left[\theta^{i}, \theta^{i+1}\right) \times\left[\theta^{j}, \theta^{j+1}\right)}(t, s) .
\end{gathered}
$$

Let us first compute the $L^{2}$-norm of $S_{d, l}(f)$. By using Plancherels theorem, Fubinis theorem, Lemma 4, plus the approaches used in [7], we obtain that

$$
\begin{aligned}
& \left\|S_{d, l}(f)\right\|_{L^{2}\left(\mathbf{R}^{n} \times \mathbf{R}^{m}\right)}^{2} \\
\leq & \left.\sum_{i, j \in \mathbf{Z}_{\Delta_{i+d, j+l}}} \int_{\theta^{i}} \int_{\theta^{i+1}}^{\theta^{j+1}} \int_{\theta^{j}}\left|\hat{\sigma}_{\Omega, \phi, \psi, h, t, s}(\xi, \eta)\right|^{2} \frac{d t d s}{t s}|| \hat{f}(\xi, \eta)\right|^{2} d \xi d \eta \\
\leq & C_{p} \ln ^{2}(\theta)\|h\|_{\Delta_{\gamma}\left(\mathbf{R}^{+} \times \mathbf{R}^{+}\right)}\|\Omega\|_{L^{q}\left(\mathbf{S}^{n-1} \times \mathbf{S}^{m-1}\right)}^{2} \\
\times & \sum_{i, j \in \mathbf{Z}} \int_{\Delta_{i+d, j+l}}\left|\xi \theta^{j d_{1}}\right|^{ \pm \frac{\alpha}{\omega q^{\prime}}}\left|\eta \theta^{j d_{2}}\right|^{ \pm \frac{\alpha}{\omega q^{\prime}}}|\hat{f}(\xi, \eta)|^{2} d \xi d \eta \\
\leq & C_{p} \ln ^{2}(\theta) 2^{-\alpha(|l|+|d|)}\|h\|_{\Delta_{\gamma}\left(\mathbf{R}^{+} \times \mathbf{R}^{+}\right)}^{2}\|\Omega\|_{L^{q}\left(\mathbf{S}^{n-1} \times \mathbf{S}^{m-1}\right)}^{2} \\
& \sum_{i, j \in \mathbf{Z}} \int_{\Delta_{i+d, j+l}}|\hat{f}(\xi, \eta)|^{2} d \xi d \eta \\
\leq & C_{p}\left(\frac{A(\gamma)}{q-1}\right)^{2} 2^{-\alpha(|l|+|d|)}\|h\|_{\Delta_{\gamma}\left(\mathbf{R}^{+} \times \mathbf{R}^{+}\right)}^{2}\|\Omega\|_{L^{q}\left(\mathbf{S}^{n-1} \times \mathbf{S}^{m-1}\right)}^{2}\|f\|_{L^{2}\left(\mathbf{R}^{n} \times \mathbf{R}^{m}\right)}^{2},
\end{aligned}
$$


where $\Delta_{i, j}=\left\{(\xi, \eta) \in \mathbf{R}^{n} \times \mathbf{R}^{m}:(|\xi|,|\eta|) \in \mathcal{I}_{i, d_{1}} \times \mathcal{I}_{j, d_{2}}\right\}$.

Now, let us compute the $L^{p}$-norm of $S_{d, l}(f)$ for any $p$ satisfying $|1 / p-1 / 2|<$ $\min \left\{1 / \gamma^{\prime}, 1 / 2\right\}$ with $p \neq 2$. By using Lemma 8, and applying the LittlewoodPaley theory and Theorem 3 along with the remark that follows its statement in [[27], pp. 96], we obtain

$$
\begin{aligned}
\left\|S_{d, l}(f)\right\|_{L^{p}\left(\mathbf{R}^{n} \times \mathbf{R}^{m}\right)} & \\
& \leq C_{p} \frac{A(\gamma)}{q-1}\|h\|_{\Delta_{\gamma}\left(\mathbf{R}^{+} \times \mathbf{R}^{+}\right)}\|\Omega\|_{L^{q}\left(\mathbf{S}^{n-1} \times \mathbf{S}^{m-1}\right)}\|f\|_{L^{p}\left(\mathbf{R}^{n} \times \mathbf{R}^{m}\right)} .
\end{aligned}
$$

Interpolation between the last inequality and (13), we deduce that there exists $0<\kappa<1$ such that

$$
\begin{aligned}
& \left\|S_{d, l}(f)\right\|_{L^{p}\left(\mathbf{R}^{n} \times \mathbf{R}^{m}\right)} \\
& \leq C_{p} \frac{A(\gamma)}{q-1} 2^{-\frac{\kappa}{2}(|l|+|d|)}\|h\|_{\Delta_{\gamma}\left(\mathbf{R}^{+} \times \mathbf{R}^{+}\right)}\|\Omega\|_{L^{q}\left(\mathbf{S}^{n-1} \times \mathbf{S}^{m-1}\right)}\|f\|_{L^{p}\left(\mathbf{R}^{n} \times \mathbf{R}^{m}\right)},
\end{aligned}
$$

holds for any $p$ with $|1 / p-1 / 2|<\min \left\{1 / \gamma^{\prime}, 1 / 2\right\}$. Consequently, by (12) and (14), we finish the proof of Theorem 1.

\section{References}

[1] H. Al-Qassem, Rough Marcinkiewicz integral operators on product spaces, Collect. Math., 56, No. 3 (2005), 275-297.

[2] H. Al-Qassem, A. Al-Salman, A note on Marcinkiewicz integral operators, J. Math. Anal. Appl., 282, No. 2 (2003), 698-710.

[3] H. Al-Qassem, A. Al-Salman, L. Cheng, Y. Pan, Marcinkiewicz integrals on product spaces, Studia Math., 167, No. 3 (2005), 227-234.

[4] H. Al-Qassem, Y. Pan, On certain estimates for Marcinkiewicz integrals and extrapolation, Collec. Math., 60, No. 2 (2009), 123-145.

[5] A. Al-Salman, H. Al-Qassem, L. Cheng, Y. Pan, $L^{p}$ bounds for the function of Marcinkiewicz, Math. Res. Lett., 9, No. 1 (2002), 697-700.

[6] M. Ali, $L^{p}$ estimates for Marcinkiewicz integral operators and extrapolation, Journal of Inequalities and Applications (2014). doi: 10.1186/1029242X-2014-269. 
[7] M. Ali, $L^{p}$ estimates for Marcinkiewicz integrals on product spaces, Houston J. of Math., accepted.

[8] M. Ali, E. Abo-Shgair, On certain estimates for parabolic Marcinkiewicz integral and extrapolation, Int. J. of Pure and App. Math., accepted.

[9] M. Ali, M. Al-Jararhah, On certain estimates for Marcinkiewicz integrals with a rough kernel on product spaces, submitted.

[10] M. Ali, M. Bataineh, $L^{p}$ boundedness for Marcinkiewicz integrals and Extrapolation, submitted.

[11] Y. Chen, Y. Ding, $L^{p}$ bounds for the parabolic Marcinkiewicz integral with rough kernels, J. Korean Math. Soc., 44, No. 3 (2007), 733-745.

[12] Y. Chen, Y. Ding, The parabolic Littlewood-Paley operator with Hardy space kernel, Canad. Math. Bull., 52, No. 4 (2009), 521-534.

[13] J. Chen, Y. Ding, D. Fan, Certain square functions on product spaces, Math. Nachr., 230, No. 1 (2001), 5-18.

[14] J. Chen, D. Fan, Y. Ying, Rough Marcinkiewicz integrals with $L(\log L)^{2}$ kernels, Adv. Math. (China), 30, No. 2 (2001), 179-181.

[15] J. Chen, D. Fan, Y. Ying, The method of rotation and Marcinkiewicz integrals on product domains, Studia Math., 153, No. 1 (2002), 41-58.

[16] Y. Choi, Marcinkiewicz integrals with rough homogeneous kernel of degree zero in product domains, J. Math. Anal. Appl., 261, No. 1 (2001), 53-60.

[17] Y. Ding, $L^{2}$-boundedness of Marcinkiewicz integral with rough kernel, Hokk. Math. J., 27, No. 1 (1998), 105-115.

[18] Y. Ding, On Marcinkiewicz integral, Proc. of the conference Singular integrals and related topics III, Oska, Japan (2001).

[19] Y. Ding, D. Fan, Y. Pan, On the $L^{p}$ boundedness of Marcinkiewicz integrals, Mich. Math. J., 50, No. 1 (2002), 17-26.

[20] J. Duoandikoetxea, J. Rubio de Francia, Maximal functions and singular integral operators via Fourier transform estimates, Invent. Math. 84, No. 3 (1986), 541-561.

[21] D. Fan, Y. Pan, A Singular integral operator with rough kernel, Proce. Amer. Math. Soci., 125, No. (1997), 3695-3703. 
[22] D. Fan, Y. Pan, Singular integral operators with rough kernels supported by subvarieties, Amer. J. Math., 119, No. 4 (1997), 799-839.

[23] L. Hörmander, Estimates for translation invariant operators in $L^{p}$ space, Acta Math., 104 (1960), 93-139.

[24] M. Sakamota, K. Yabuta, Boundedness of Marcinkiewicz functions, Studia Math., 135, No. 2 (1999), 103-142.

[25] E. Stein, Harmonic Analysis: Real-Variable Methods, Orthogonality and Oscillatory Integrals, Princeton University Press, Princeton, NJ, 1993.

[26] E. Stein, On the functions of Littlewood-Paley, Lusin and Marcinkiewicz, Trans. Amer. Math. Soc., 88 (1958), 430-466.

[27] E. Stein, Singular integrals and differentiability properties of functions, Princeton University Press, Princeton, NJ, 1970.

[28] T. Walsh, On the function of Marcinkiewicz, Studia Math., 44, No. 3 (1972), 203-217.

[29] H. Wu, Boundedness of multiple Marcinkiewicz integral operators with rough kernels. J. Korean. Math. Soc., 43, No. 3 (2006), 635-658.

[30] H. Wu, General Littlewood-Paley functions and singular integral operators on product spaces, Math. Nachr., 279, No. 4 (2006), 431-444.

[31] Y. Wu and H. Wu, A Note on the Generalized Marcinkiewicz Integral Operators with Rough Kernels, Acta Math. Sin., 28, No. 12 (2012), 23952406. 